\title{
Field-dependent Mechanism of Anomalous Proton Conductivity and the Polarizability of Hydrogen Bonds with Tunneling Protons
}

\author{
E. G. WEIDEMANN \\ Sektion Physik der Universität München \\ und G. ZunDEL \\ Physikalisch-Chemisches Institut der Universität München \\ (Z. Naturforsch. 25 a, 627-634 [1970] ; received 29 December 1969)

\begin{abstract}
The displacement of the excess charge of the proton in acid solutions is caused by a structure migration of groupings $\mathrm{H}_{5} \mathrm{O}_{2}{ }^{+}$or $\mathrm{H}_{9} \mathrm{O}_{4}{ }^{+}$. The processes which take place during structure migration are discussed on the basis of results gained in IR-investigations. In an electrical field the structure migration is given a preferred direction. The hydrogen bond with the tunneling proton in $\mathrm{H}_{5} \mathrm{O}_{2}{ }^{+}$ and the grouping $\mathrm{H}_{3} \mathrm{O}^{+}$in $\mathrm{H}_{5} \mathrm{O}_{2}{ }^{+}$become polarized. Comparison of both polarizabilities demonstrates that, contrary to previous assumptions, the polarization of the hydrogen bond is the field-dependent mechanism. This conclusion is reached upon calculating the polarizability of the hydrogen bond with a symmetrical double minimum potential well. It is shown that the polarizability is extremely large, being approximately two orders of magnitude greater than that of $\mathrm{H}_{3} \mathrm{O}^{+}$. Despite the large polarizability, the shift of the weights of the proton boundary structures is very small for the external fields usually applied in conductivity measurements. It is demonstrated, however, that this slight shift is large enough for the structure diffusion to obtain a preferred direction consistent with the anomalous high proton conductivity.
\end{abstract}

\section{Mechanism of Anomalous Proton Conductivity}

The nature of the excess proton hydrate structure has been clarified ${ }^{1-4}$ by the example of polystyrene sulfonic acid by means of IR-spectroscopic investigations. With a small degree of hydration groupings $\mathrm{H}_{5} \mathrm{O}_{2}{ }^{+}$form, whereas with a large degree of hydration groupings $\mathrm{H}_{9} \mathrm{O}_{4}{ }^{+}$play a special role within the hydrate structure network. The special role of groupings $\mathrm{H}_{9} \mathrm{O}_{4}^{+}$has been demonstrated in numerous publications with the most diverse methods. Reviews of these papers are given in Refs. ${ }^{4-6}$. A symmetrical potential well with a double minimum, in which the excess proton tunnels, is present in the hydrogen bridge of grouping $\mathrm{H}_{5} \mathrm{O}_{2}{ }^{+}$. This grouping can thus be described by both proton boundary

Sonderdruckanforderungen an Priv.-Doz. Dr. E. G. WEIDEManN, Sektion Physik, D-8000 München 13, Schellingstr. $2-8$ (III)

1 G. Zundel and H. Metzger, Z. Phys. Chem. Frankfurt 58, 225 [1968].

2 G. Zundel and H. Metzger, Z. Phys. Chem. Frankfurt 59, 225 [1968].

3 G. Zundel and H. Metzger, Z. Naturforsch. 22 a, 1412 [1967].

4 G. Zundel, Hydration and Intermolecular Interaction, Academic Press, New York 1969.

5 H. L. Clever, J. Chem. Education 40, 637 [1963].

6 M. Eigen, Angew. Chem. 75, 489 [1963] ; internat. ed. 3, 1 [1964]. structures, shown on the left in Fig. 1. These groupings are linked via hydrogen bridges with other water molecules or anion acceptor groups. These hydrogen bridges are very often ruptured during thermal rearrangement processes in the hydrate structure $^{7-11}$. IR-spectroscopic findings, however, show that the number of the free $\mathrm{OH}$ groups, i. e., those not bound by hydrogen bridges, is small at room temperature ${ }^{4,12}$ and ${ }^{13}$. The charge migrates when groupings $\mathrm{H}_{5} \mathrm{O}_{2}^{+}$or $\mathrm{H}_{9} \mathrm{O}_{4}^{+}$, respectively, shift within the hydrate structure network. This takes place such that the proton tunneling in $\mathrm{H}_{5} \mathrm{O}_{2}{ }^{+}$exchanges its role as excess proton with one of the $\mathrm{H}$-nuclei from the two water molecules in $\mathrm{H}_{5} \mathrm{O}_{2}{ }^{+}$. The migration of one grouping of size $\mathrm{H}_{5} \mathrm{O}_{2}{ }^{+}$is sufficient for charge transport.

7 T. J. Swift and R. E. Connick, J. Chem. Phys. 37, 307 [1962].

8 H. G. Hertz and M. D. Zeidler, Ber. Bunsenges. Phys. Chem. 67, 774 [1963].

9 H. G. Hertz and M. D. Zeidler, Ber. Bunsenges. Phys. Chem. 68, 821 [1964].

10 M. Eigen, Pure Appl. Chem. 6, 97 [1963].

11 D. G. Smith and J. G. Powles, J. Mol. Phys. 10, 451 [1966].

12 W. A. P. Luck, Ber. Bunsenges. Phys. Chem. 69, 626 [1965].

13 T. T. WALl and D. F. Hornig, J. Chem. Phys. 43, 2079 [1965]. 
The rate-determining step of the migration of the excess protons in acid solutions is, as shown especially by EIGEN and co-workers ${ }^{6,14}, 15$, not the tunneling of the excess proton in the hydrogen bridges of $\mathrm{H}_{5} \mathrm{O}_{2}^{+}$or $\mathrm{H}_{9} \mathrm{O}_{4}^{+}$, but rather the structure diffusion of the groupings with the tunneling proton. That is, the proton tunnels frequently to and fro in the hydrogen bridge of the $\mathrm{H}_{5} \mathrm{O}_{2}{ }^{+}$until structure diffusion of this grouping ensues.

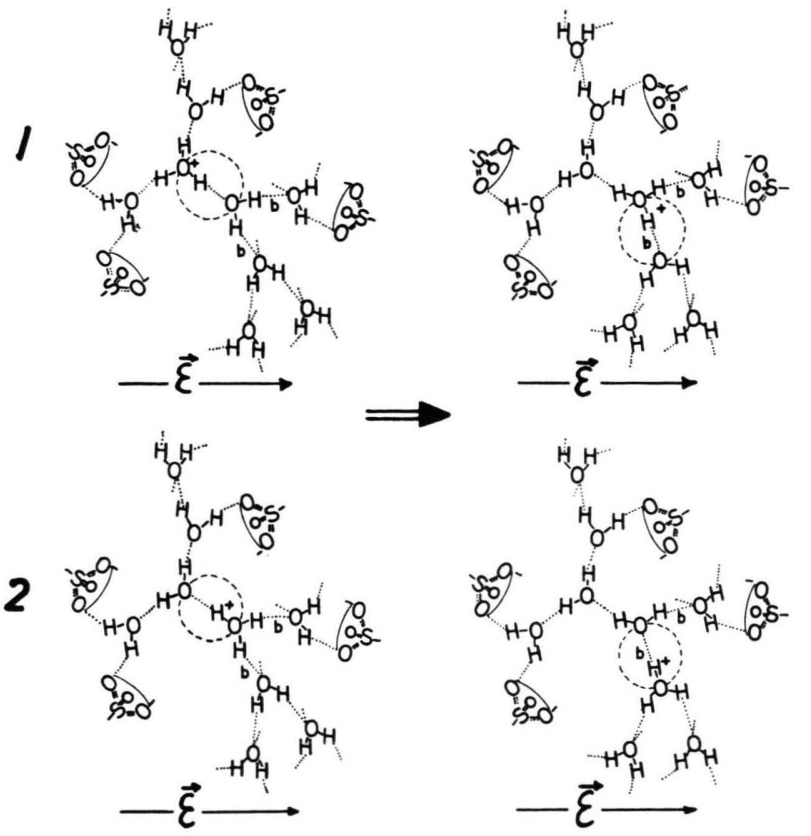

Fig. 1. Left side: two proton boundary structures of the excess proton in the $\mathrm{H}_{5} \mathrm{O}_{2}{ }^{+}$group in polysterene sulfonic acid, right side: two proton boundary structures of the excess proton after one step of structure diffusion. - In considering such schematic representations, we should keep in mind the fact that the solvate structures are continually being rearranged by thermal motions ${ }^{7-11}$. Any attachment position is in dynamic equilibrium with analogous temporary equilibrium configurations.

The processes during structure diffusion are as follows: When the excess proton is in the boundary position, shown by boundary structure 2 on the left of Fig. 1, each of the three $\mathrm{H}$-nuclei of $\mathrm{H}_{3} \mathrm{O}^{+}$can take over the role of the excess proton, i. e., a state of almost triple degeneracy with respect to the excess proton occurs for $\mathrm{H}_{3} \mathrm{O}^{+}$. The degeneracy, however, is not complete, because bridges $b$ will normally be bent, resulting in the fact that the $\mathrm{H}$ nucleus in these bridges can tunnel only with dif-

14 M. Eigen and L. De Maeyer, Proc. Roy. Soc. London A 247, 505 [1958]. ficulty, since the potential well although largely symmetrical, possesses a relatively high barrier. If one of the bridges $b$ becomes linearized during thermal movement, this bridge will no longer be distinguishable from the one in $\mathrm{H}_{5} \mathrm{O}_{2}{ }^{+}$. A doubly degenerate state with respect to the excess proton will therefore occur for grouping $\mathrm{H}_{3} \mathrm{O}^{+}$. When the $\mathrm{H}$-nucleus now tunnels in bridge $\mathrm{b}$ and when the bridge in which the excess proton has previously tunneled is finally bent, structure diffusion has taken place. The comparison of both proton boundary structures on the left of Fig. 1 with the two proton boundary structures on the right illustrates this process.

Why is this process given a preferred direction when an external electrical field is applied? Two causes are conceivable:

a) By means of the external field, the weight of the proton boundary structure, in which the excess proton lies in the field direction (2 on the left in Fig. 1), can be increased. This increases the probability that the excess proton changes roles with one of the protons in the hydrogen bridges $b$, as soon as these are linearized. Thus structure migration and also the charge transport occur preferably in field direction.

b) The field-dependent mechanism could be the polarization of $\mathrm{H}_{3} \mathrm{O}^{+}$, for we have seen that a doubly degenerate state with respect to the excess proton occurs for $\mathrm{H}_{3} \mathrm{O}^{+}$when one of the bridges $\mathrm{b}$ is considerably linearized. This degeneracy is removed by the external field, since that state is preferred for $\mathrm{H}_{3} \mathrm{O}^{+}$whose excess charge lies at the $\mathrm{H}$-nucleus in bridge $\mathrm{b}$ in field direction. The migration of the structure with the excess proton in the opposite field direction is likewise prevented. This causes structure diffusion to occur preferably in the field direction.

The question as to whether a) or b) is the fielddependent mechanism of anomalous proton conductivity can be resolved by comparing the polarizability of the bridge in $\mathrm{H}_{5} \mathrm{O}_{2}^{+}$with that of $\mathrm{H}_{3} \mathrm{O}^{+}$. The former can be estimated by a simple calculation.

In this calculation the interaction between the tunneling protons by proton dispersion forces ${ }^{4}$ as well as the influence of the anion fields is neglected. Thus the results are valid in the case of infinite dilution only.

15 M. Eigen, Z. Elektrochem. 64, 115 [1960]. 


\section{Energy Levels of a Hydrogen Bridge with a Symmetrical Double Minimum Potential Well in an Electrical Field}

We consider here a single hydrogen bridge with a symmetrical double minimum potential in a static electrical field. Let us assume that the bridge lies in $x$-direction with its center at the origin of the coordinate system. $\mathcal{E}$ is the component of the electrical field strength in the positive $x$-direction. If $e$ is the tunneling proton charge, or the displaced effective charge taking the polarization of the electron cloud into consideration, respectively, the interaction energy of the proton and the electrical field is

$$
W=-e \varepsilon x .
$$

Fig. 2 shows the energy levels of the tunneling proton. The levels 0 and 1 , respectively, are split by the tunneling into two states each of positive and nega-

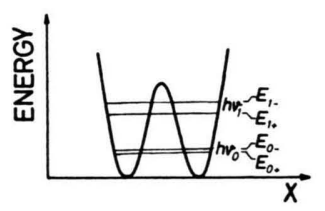

Fig. 2. Symmetrical double minimum potential well in a hydrogen bond.

tive parity. $v_{0}$ is the tunneling frequency. These levels will shift under the influence of the interaction energy $W$ and the stationary states in the given external field can be investigated. These give information on the weight of the proton boundary structures in or opposite to the field direction.

We restrict ourselves to small fields so that the shift of both lower levels $0+$ and 0 - is small compared to the distance between levels 0 and 1 . Numerical evaluation shows that this assumption is extremely well fulfilled for the fields under discussion. Thus we can neglect transitions to the upper levels and represent the stationary states $\varphi_{ \pm}$in the field $\mathcal{E}$ as a superposition of states $\psi_{0+}$ and $\psi_{0-}$.

$$
\varphi_{ \pm}=c_{+} \psi_{0+}+c_{-} \psi_{0_{-}} .
$$

Substituting this expansion in the eigenvalue equation

$$
H \varphi=E \varphi
$$

and multiplying from the left by $\psi_{0+}^{*}$ and integrating,

$$
\begin{aligned}
& \int \psi_{0+}^{*} H \psi_{0+} \mathrm{d} x c_{+}+\int \psi_{0+}^{*} H \psi_{0-} \mathrm{d} x c_{-} \\
& =E\left[\int \psi_{0+}^{*} \psi_{0+} \mathrm{d} x c_{+}+\int \psi_{0+}^{*} \psi_{0-} \mathrm{d} x c_{-}\right]
\end{aligned}
$$

results. The right side of this equation becomes $E c_{+}$on account of the normalization and the orthogonality of the undisturbed eigenfunctions. A corresponding equation results on multiplying by $\psi_{0^{-}}^{*}$, Both equations can be abbreviated as

$$
\begin{aligned}
& H_{++} c_{+}+H_{+_{-}} c_{-}=E c_{+}, \\
& H_{-+} c_{+}+H_{--} c_{-}=E c_{-} .
\end{aligned}
$$

The diagonal elements of the Hamiltonian $H_{+}+$ and $H_{z_{-}}$are the energy levels without an electrical field which lie at $\pm \frac{1}{2} h v_{0}$ when the mid-point of both lower levels $0+$ and $0-$ is chosen as the energy zero point.

$$
H_{++}=-\frac{1}{2} h v_{0}, H_{--}=+\frac{1}{2} h v_{0} .
$$

These expressions follow because $H$ is composed additively of $H^{0}$, the Hamiltonian without a field, whose eigenvalues are given in Fig. 2, and the interaction $W$ with the electrical field. Hence

$$
\begin{aligned}
H_{++}=H_{++}^{0} & +W_{++}=\int \psi_{0+}^{*}(x) H^{0} \psi_{0+}(x) \mathrm{d} x \\
& +\int \psi_{0+}^{*}(x) W \psi_{0+}(x) \mathrm{d} x .
\end{aligned}
$$

$\psi_{0+}$ is the eigenfunction of $H^{0}$ for the eigenvalue $-\frac{1}{2} h v_{0}$, hence $H_{++}^{0}=-\frac{1}{2} h v_{0} \cdot W_{++}$disappears, since $W$ has matrix elements which only differ from zero between states of different parity.

This is demonstrated when the integration variable $x$ is replaced by $-x$.

$W_{++}=-e \varepsilon \int \psi_{0_{+}}^{*}(x) x \psi_{0+}(x) \mathrm{d} x$

$$
=-e \varepsilon \int \psi_{0+}^{*}(-x)(-x) \psi_{0+}(-x) \mathrm{d} x .
$$

Hence, $W_{++}$is equal to $+e \varepsilon \int \psi_{0+}^{\star}(x) x \psi_{0-}(x) \mathrm{d} x$, because $\psi_{0+}(-x)=\psi_{0+}(x)$. From $W_{++}=-W_{++}$it follows $W_{++}=\mathbf{0}$.

Corresponding considerations apply for the second diagonal element $H_{-} \ldots$.

$H^{0}$ does not contribute to the non-diagonal elements, since

$H_{+-}^{0}=\int \psi_{0+}^{*} H^{0} \psi_{0-} \mathrm{d} x=+\frac{1}{2} h v_{0} \int \psi_{0+}^{*} \psi_{0-} \mathrm{d} x=0$

on account of the orthogonality of the eigenfunctions of $H^{0}$. These matrix elements

$$
H_{+-}=W_{+-}=-\varepsilon e \int \psi_{0+}(x) x \psi_{0-}(x) \mathrm{d} x=H_{-+}
$$

can be expressed by the transition dipole moment introduced previously [Ref. ${ }^{16} \mathrm{cf}$. also ${ }^{4}$ ] where real eigenfunctions are chosen

$$
p=e \int \psi_{0+}(x) x \psi_{0_{-}}(x) \mathrm{d} x .
$$

16 E. G. Weidemann and G. Zundel, Z. Physik 198, 288 [1967]. 
Hence it follows that

$$
H_{+-}=H_{-+}=-\varepsilon p .
$$

Eq. (5) together with Eq. (6) and (12) gives

$$
\begin{aligned}
& \left(-\frac{1}{2} h v_{0}-E\right) c_{+}-\varepsilon p c_{-}=0, \\
& -\varepsilon p c_{+}+\left(\frac{1}{2} h v_{0}-E\right) c_{-}=0,
\end{aligned}
$$

and from this the secular equation

$$
\left|\begin{array}{cc}
-\frac{1}{2} h v_{0}-E & -\varepsilon p \\
-\varepsilon p & \frac{1}{2} h v_{0}-E
\end{array}\right|=0,
$$

which yields the eigenvalues

$$
\begin{array}{r}
E_{0 \pm}=\mp \sqrt{\left(\frac{1}{2} h v_{0}\right)^{2}+(\varepsilon p)^{2}}=\mp \frac{1}{2} h v_{0} \sqrt{1+Z^{2}}, \\
\\
Z=2 \varepsilon p / h v_{0} .
\end{array}
$$

The levels are shifted in the field $\varepsilon$ in the manner shown in Fig. 3.

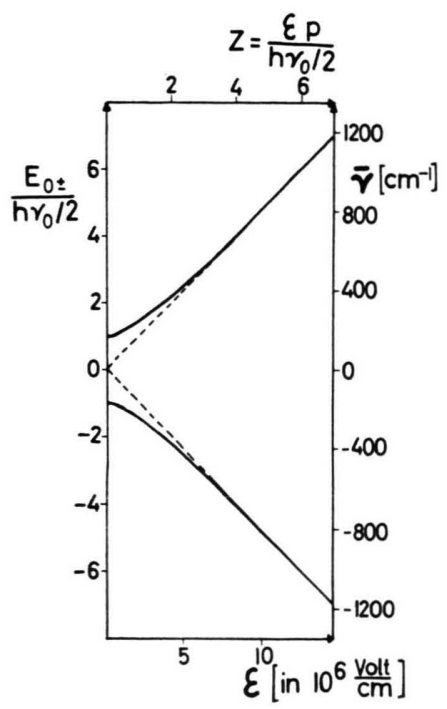

Fig. 3. The dependence of the shift of the energy levels in a hydrogen bridge with a tunneling proton on the electrical field.

For large values of the parameter $Z, E_{0 \pm}$ is approximately $\mp \varepsilon p$, i. e., the potential energy of a dipole $\mathrm{p}$ which is aligned in the ground state $0+$ parallel to the field direction and in the state $0-$ antiparallel to the field direction.

The second scales in Fig. 3 give the shifts of the energy levels in wave numbers dependent on the field for the special case

$$
v_{0}=10^{13} \sec ^{-1} \text { and } p=2 \AA \cdot \frac{1}{2} e .
$$

The value of $v_{0}$ is given by EIGEN ${ }^{6}$. In this paper $(0.8 \div 1) \times 10^{-13} \mathrm{sec}$ is given as the lifetime of $\mathrm{H}_{3} \mathrm{O}^{+}$in ice. The reciprocal of this value corresponds to the tunneling frequency in $\mathrm{H}_{5} \mathrm{O}_{2}{ }^{+}$or
$\mathrm{H}_{9} \mathrm{O}_{4}{ }^{+}$, respectively. The choice of this value $p$ is explained in detail in ${ }^{4,16}$. It can be seen that the shift of energy levels for fields, as they usually occur in solutions, is extremely small. This already gives rise to the presumption that the weights of the proton boundary structures also change only minimally.

\section{Shift of Weights of Proton Boundary Structures}

These weights result from the expansion coefficients $c_{+}$and $c_{-}$which can be calculated from Eq. (13). If we choose the phases of the stationary wave functions without a field $\psi_{0+}$ and $\psi_{0-}$ as in Fig. 4, the states

$\psi_{\mathrm{r}}=\frac{1}{\sqrt{2}}\left(\psi_{0+}+\psi_{0_{-}}\right), \psi_{\mathrm{l}}=\frac{1}{\sqrt{2}}\left(\psi_{0_{+}}-\psi_{0_{-}}\right)$

correspond to the boundary structures whereby the proton is shifted completely to the right (in field direction) or to the left (opposite to field direction), respectively (see Fig. 4). We can now also expand the stationary states in the electrical field in terms of the states $\psi_{\mathrm{r}}$ and $\psi_{\mathrm{l}}$,

$$
\varphi_{ \pm}=\boldsymbol{a}_{\mathrm{r}} \psi_{\mathrm{r}}+a_{1} \psi_{\mathrm{l}},
$$
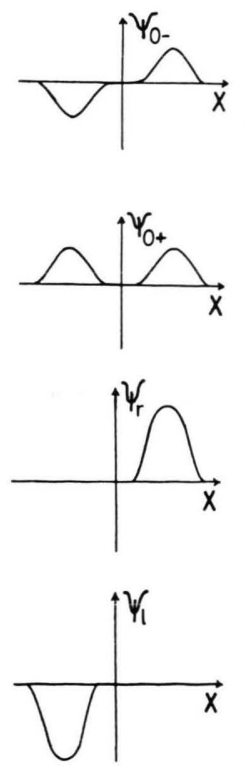

Fig. 4. a) The states $\psi_{0+}$ and $\psi_{0}$. b) The states $\psi_{\mathrm{r}}$ and $\psi_{\mathrm{l}}$.

instead of as in Eq. (2) in terms of the states $\psi_{0+}$ and $\psi_{0-}$. Substituting Eq. (16) and comparing with Eq. (2) we find the following relations between the 
expansion amplitudes

$$
c_{+}=\frac{1}{\sqrt{2}}\left(a_{\mathrm{r}}+a_{1}\right), \quad c_{-}=\frac{1}{\sqrt{2}}\left(a_{\mathrm{r}}-a_{1}\right),
$$

respectively, which gives

$a_{\mathrm{r}}=\frac{1}{\sqrt{2}}\left(c_{+}+c_{-}\right), \quad a_{1}=\frac{1}{\sqrt{2}}\left(c_{+}-c_{-}\right)$.

The weights of the boundary structures preferred or impaired by the field are

$$
G_{\mathrm{r}}=\left|a_{\mathrm{r}}\right|^{2}, \quad G_{\mathrm{l}}=\left|a_{1}\right|^{2},
$$

respectively,

so that due to Eq. (16)

$$
G_{\mathrm{r}}=\left|c_{+}+c_{-}\right|^{2}, \quad G_{1}=\left|c_{+}-c_{-}\right|^{2} .
$$

We express the charge shift in the bridge by

$$
\Delta G=G_{\mathrm{r}}-G_{\mathrm{l}} .
$$

$\Delta G$ assumes the value +1 when the proton is completely shifted in the field direction (to the right), the value 0 when both boundary structures (without field) have the same weight, and the value -1 in the case of polarization in the opposite-field direction. By Eq. (21) $\Delta G$ is calculated from the expansion amplitudes determined by Eq. (13) as

$$
\Delta G=c_{+}^{*} c_{-}+c_{-}^{*} c_{+} .
$$

In order to calculate the amplitudes, we solve the first of the two equations (13) by the ansatz

$$
c_{+}=-\varepsilon p A, \quad c_{-}=\left(\frac{1}{2} h v_{0}+E\right) A .
$$

The second Eq. (13) is thus also satisfied, since through Eq. (14) it depends linearly on the first. Constant $A$ is given by the normalization condition $\left|c_{+}\right|^{2}+\left|c_{-}\right|^{2}=1$ :

$$
A=\frac{1}{\left.\sqrt{(\varepsilon p)^{2}+\left(\frac{1}{2} h\right.} h v_{0}+E\right)^{2}} .
$$

For $\Delta G$ we obtain

$\Delta G=\left[-2 \mathcal{E} p\left(\frac{1}{2} h v_{0}+E\right)\right] /\left[(\mathcal{E} p)^{2}+\left(\frac{1}{2} h v_{0}+E\right)^{2}\right]$, or, using the abbreviation $Z$ from Eq. (15)

$$
\Delta G=\frac{-2 Z\left(1+E / \frac{1}{2} h v_{0}\right)}{Z^{2}+\left(1+E / \frac{1}{2} h v_{0}\right)^{2}} .
$$

Hence both eigenvalues from Eq. (15) are given as

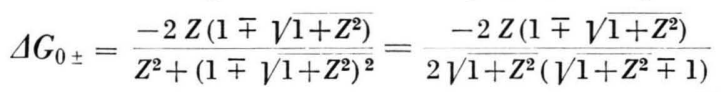

and thus

$$
\Delta G_{0 \pm}= \pm Z / \sqrt{1+Z^{2}} .
$$

This is the shift of the weights of the proton boundary structures in the electrical field. Substi- tuting as above $v_{0}=10^{13} \mathrm{sec}^{-1}$ and $p=2 \AA \cdot \frac{1}{2} e$, one obtains $\Delta G_{0 \pm}= \pm 4.8 \times 10^{-7}$ for a field strength of $1 \mathrm{Volt} / \mathrm{cm}$. That is, the shift of the weights of the proton boundary structures is extremely small in the case of field strengths as they usually occur in solutions. Therefore the surmise was expressed ${ }^{4}$ that the shift of the weights of the proton boundary structure through the field, i. e., mechanism a), cannot be the field-dependent mechanism in the case of anomalous proton conductivity. We shall see in the following, however, that such a slight shift of the weights is already sufficient to bring about the preferred direction of the structure migration through the electrical field.

\section{Induced Dipole Moment}

The characteristic quantity $\Delta G$ for the shift of the weights of the proton boundary structures is proportional to the dipole moment $\mu$ induced in the hydrogen bridge since the moment induced in the field $\mathcal{E}$ is the expectation value of the dipole operator $e \cdot x$ with respect to the stationary states in this field

$$
\mu=e \int \varphi^{*}(x) x \varphi(x) \mathrm{d} x .
$$

Substituting Eq. (2) we obtain

$\mu=e \int\left(c_{+} \psi_{0_{+}}+c_{-} \psi_{0_{-}}\right) * x\left(c_{+} \psi_{0+}+c_{-} \psi_{0_{-}}\right) \mathrm{d} x$

and as there are no dipole transitions between states of the same parity

$\mu=e \int \psi_{0_{+}}(x) x \psi_{0_{-}}(x) \mathrm{d} x\left(c_{+}^{*} c_{-}+c_{-}^{*} c_{+}\right)$.

For ground state $0+$ and the first excited state $0-$ we hence obtain, on account of Eq. (11) and Eq. (23), the induced dipole moments

$$
\mu_{0 \pm}(\varepsilon)=p \Delta G_{0 \pm}(\varepsilon) .
$$

At temperature $T$ the ground state and the first excited state are occupied according to the probabilities determined by the Boltzmann factors

$$
\begin{aligned}
w_{0 \pm}(T, \varepsilon)=\exp \left(-\frac{E_{0+}(\varepsilon)}{k T}\right) /[ & \exp \left(-\frac{E_{0+}(\varepsilon)}{k T}\right) \\
+ & \left.\exp \left(-\frac{E_{0-}(\varepsilon)}{k T}\right)\right]
\end{aligned}
$$

whereby we have neglected the remaining excited states in the partition function. This is justified as long as these states are almost totally unoccupied, i. e., as long as

$$
k T \ll E_{1+}-E_{0+} .
$$


The occupation probabilities depend on the field strength as well as on the temperature, since the energy levels (15) shifted in the field must of course be substituted. The dipole moment induced at temperature $T$ in the field $\mathcal{E}$ is therefore

$$
\mu(T, \varepsilon)=\mu_{0+}(\varepsilon) w_{0+}(T, \varepsilon)+\mu_{0-}(\varepsilon) w_{0-}(T, \varepsilon) .
$$

With Eq. (33), (34) and (15) the following results

$$
\begin{aligned}
\mu(T, \varepsilon)=p \frac{Z}{\sqrt{1}+Z^{2}} \tanh \left(\frac{h v_{0}}{2 k T} \sqrt{1+Z^{2}}\right) \\
Z=\frac{2 p}{h v_{0}} \varepsilon .
\end{aligned}
$$

In Fig. $5 \mu$ is plotted as a function of the field strength for temperatures $T=100^{\circ}, 200^{\circ}, 300^{\circ} \mathrm{K}$. If $Z \ll 1$, i. e., if $p \varepsilon \ll \frac{1}{2} h v_{0}, \mu$ is an almost completely linear function of the field strength. For large field strengths, $Z \gg 1, \mu$ approximates the saturation value $p$.

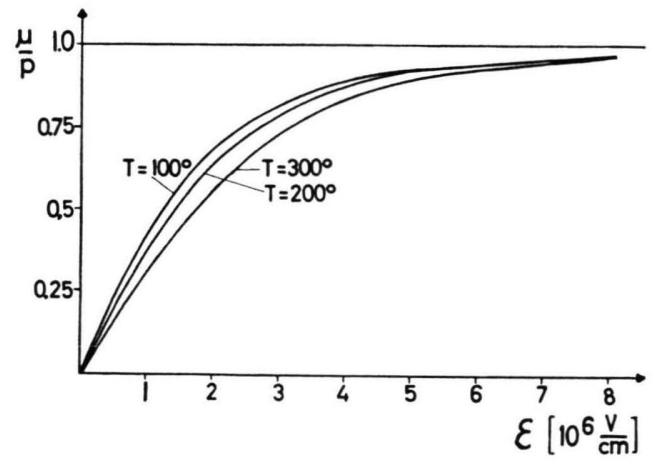

Fig. 5. The dependence of the induced dipole moment in a symmetrical hydrogen bond with tunneling proton (in units of the transition moment $p$ between the two lowest states) on the electrical field for different temperatures.

Here it must not be forgotten, however, that only states $0+$ and $0-$ were taken into consideration in the calculation. The results thus apply only as long as the shift of these terms is small compared to their distance from levels $1+$ and $1-$, i. e., as long as,

$$
\frac{1}{2} h v_{0}\left(\sqrt{1+Z^{2}}-1\right) \ll E_{1+}-E_{0-}
$$

on account of Eq. (15). This condition is compatible with $Z \ll 1$, if

$$
p \varepsilon \ll E_{1+}-E_{0-} .
$$

Due to conditions (35) and (39) the range of validity of Eq. (37) is limited to temperatures and field strengths

$$
T \ll 2 \cdot 10^{3}{ }^{\circ} \mathrm{K}, \quad \varepsilon \ll 10^{7} \mathrm{~V} / \mathrm{cm}
$$

when the numerical values $p=2 \AA \cdot \frac{1}{2} e$ and $E_{1_{+}}-E_{0+} \approx E_{1+}-E_{0-} \approx 0.2 \mathrm{eV}$ are again used. These limits to the theory are thus practically insignificant.

\section{Polarizability of a Hydrogen Bridge with a Double Minimum Potential Well}

The linear rise of the curves in Fig. 5 gives the polarizability $\alpha(T)$ of the hydrogen bridge

$$
\alpha(T)=\left.\frac{\partial \mu(T, \varepsilon)}{\partial \varepsilon}\right|_{\varepsilon=0}
$$

Differentiating Eq. (27) gives, on account of

$$
\begin{gathered}
\left.\frac{\partial \mu}{\partial \varepsilon}\right|_{\varepsilon=0}=\left.\frac{2 p}{h v_{0}} \frac{\partial \mu}{\partial Z}\right|_{Z=0}, \\
\alpha(T)=2 p^{2} / h v_{0} \tanh \left(h v_{0} / 2 k T\right) .
\end{gathered}
$$

The dependence of the polarizability on the tunneling frequency $v_{0}$ at temperatures $T=100^{\circ}, 200^{\circ}$, $300{ }^{\circ} \mathrm{K}$ is represented in Fig. 6 . In the case of small

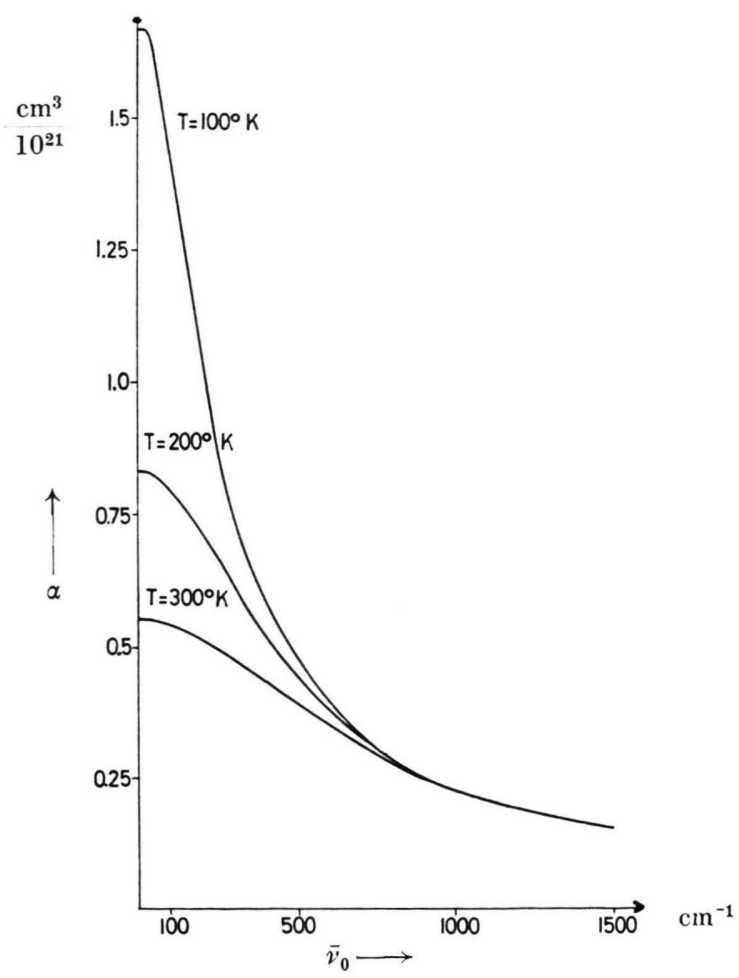

Fig. 6. The dependence of the polarizability of a symmetrical hydrogen bond with tunneling proton on the tunneling frequency in $\mathrm{cm}^{-1}$.

tunneling frequencies, $\alpha$ assumes the value $p^{2} / k T$ independent of $\nu_{0}$. With large tunneling frequencies $\alpha$ behaves like $2 p^{2} / h v_{0}$. $\alpha$ decreases steadily with $v_{0}$, since Eq. (42) can also be expressed as

$$
\alpha(T)=\frac{p^{2}}{k T} \frac{\tanh y}{y}, \quad y=\frac{h v_{0}}{2 k T} .
$$

Furthermore, it can be seen from Eq. (43) that the maximum decreases inversely proportionally to the 
temperature and the half-width values of the curves rise proportionally to $T$.

For a tunneling frequency of $10^{13} \mathrm{sec}^{-1}$ and $p=2 \AA \cdot \frac{1}{2} e\left(2 p^{2} / h v_{0}=6.96 \times 10^{-22} \mathrm{~cm}^{3}\right)$ at room temperature, it follows that

$$
\alpha=4.62 \times 10^{-22} \mathrm{~cm}^{3} .
$$

The fact that the maximum polarizability according to Eq. (43) is reached at $v_{0}=0$ indicates a limit to the theory that has not been mentioned so far. In the calculation it is assumed that a stationary state has been achieved in the field and, in addition, the thermal equilibrium with the environment has been reached, as assumed in Eq. (34). The reciprocal tunneling frequency $1 / v_{0}$ is a measure of the attainment of the stationary state, at least some transitions between the proton boundary structures must have taken place. Thus, a stationary state can no longer be attained in the event that $1 / v_{0}$ is comparable with the lifetime $\tau$ of the hydrogen bridges, hence

$$
v_{0} \gg 1 / \tau
$$

must be assumed to ensure the validity of Eq. (43). The relaxation time necessary for the occupation of levels $E_{0+}$ and $E_{0-}$ to reach thermal equilibrium, however, involves no lower limit restriction on the frequency, since the equilibrium distribution between the two lowest levels should be reached the faster, the less their distance.

\section{Determination of the Field-dependent Mechanism}

By comparing the polarizability of the hydrogen bridges with tunneling proton in $\mathrm{H}_{5} \mathrm{O}_{2}{ }^{+}$with the polarizability of $\mathrm{H}_{3} \mathrm{O}^{+}$it can now be decided whether mechanism a), i. e., the shift of the weights of the proton boundary structures, or mechanism b), i. e., the polarization of $\mathrm{H}_{3} \mathrm{O}^{+}$, is the field-dependent mechanism in the case of anomalous proton conductivity. The polarizability for free $\mathrm{H}_{3} \mathrm{O}^{+}$ions is according to ${ }^{17}$

$$
\alpha_{\mathrm{H}_{3} 0+}=1.2 \times 10^{-24} \mathrm{~cm}^{3}
$$

whereas the polarizability of the hydrogen bridge with a tunneling proton is of the order of magnitude $10^{-22} \mathrm{~cm}^{3}$, i. e., the polarizability of the hydrogen bridges with a tunneling proton is two orders of magnitude greater than that of $\mathrm{H}_{3} \mathrm{O}^{+}$. Accordingly, the influence of the field on the hydrogen bridge is the field-dependent mechanism.

This great polarizability arises because the ground state is split by the tunneling of the proton. Therefore, an excited state with a very small excitation

\footnotetext{
17 K. Fajans and G. Joos, Z. Physik 23, 1 [1924].
}

energy is present which has odd parity in contrast to the ground state. Thus, only a small electrical field is necessary to admix this state and cause an asymmetric charge distribution. This influence consists in shifting the weights of the proton boundary structures. This shift, however, is extremely small, as we know. For this reason it has even been assumed previously ${ }^{4}$ that it cannot be of great significance, as far as proton conductivity is concerned. The following estimate, however, shows that the slight shift of the weights affects structure diffusion in the field direction in such a favorable way that anomalous proton conductivity is brought about.

As mentioned above structure diffusion is the rate determining step in anomalous proton conductivity in acid solutions ${ }^{6}$. Without an external field, $\nu_{\mathrm{D}}$ shall be the number of structure diffusion steps per second without giving preference to any direction in space. The mean distance $s$ covered by a diffusion step * is approximately $2.5 \AA$. In an electrical field the frequency of diffusion steps in the field direction is equal to $v_{\mathrm{D}}$ times the probability of the proton boundary structure in which the proton is displaced in the field direction. The corresponding consideration applies to the migration in the opposite field direction. Thus the difference in the movement in and opposite to the field direction is proportional to $v_{\mathrm{D}} \Delta G$, if the hydrogen bridge containing the excess proton prior to the diffusion step lies parallel to the field. Upon averaging $s \Delta G$ over all orientations of the hydrogen bridges, the mean velocity of the protons is

$$
\bar{v}=v_{\mathrm{D}} \overline{s \Delta G} .
$$

According to Eq. (29), for fields usually applied in conductivity measurements, $\Delta G$ is proportional with a high degree of accuracy to the field strength. If $\vartheta$ is the angle between the hydrogen bridge and the field direction, only the component $\varepsilon \cos \vartheta$ becomes effective in polarization and only the component

\footnotetext{
* With the structure migration of the $\mathrm{H}_{5} \mathrm{O}_{2}{ }^{+}$or $\mathrm{H}_{9} \mathrm{O}_{4}^{+}$grouping the linearization of the hydrogen bridge connecting the external water molecules with their environment is - as discussed above - of decisive importance. The potential barrier in the double minimum potential well becomes smaller as the bridges become more linear. Thus, the tunneling frequency decreases as the linearization of the bridges increases. Since the polarizability of the bridges is dependent on the tunneling frequency (Fig. 6), the structure diffusion rate, i. e. $v_{\mathrm{D}}$, is likewise influenced by the field to some extent. In the following estimate this influence is negligible, since the dependence is of no relevance for small fields, as can be seen from Fig. 6 .
} 
$s \cos \vartheta$ is covered by one diffusion step, so that

$$
\overline{s \Delta G}=s \frac{\mu}{p} \overline{\cos ^{2} \vartheta}=\frac{1}{3} s \frac{\mu}{p}=\frac{1}{3} s \frac{\alpha}{p} \varepsilon .
$$

It can easily be verified that $\overline{\cos ^{2} \vartheta}$ amounts to $\frac{1}{3}$ by averaging over all orientations of the hydrogen bridge, i. e., over $0<\vartheta<\pi / 2$ and azimuth $\varphi$ over $0<\varphi<2 \pi . \alpha$ is given by Eq. (42) whereby the factor $\tanh \left(h v_{0} / 2 k T\right)$ takes the thermal occupation of the $0-$ level into account. As $\alpha$ is dependent on $v_{0}$ the mean velocity is influenced by the tunneling frequency, although structure diffusion is the rate determining step. The mobility $u=\lambda_{0} / F=3.5 \times 10^{-3}$ $\mathrm{cm}^{2} /$ Volt $\cdot$ sec defined by

$$
\bar{v}=u \varepsilon
$$

is obtained from conductivity measurements. $\lambda_{0}$ is the limiting equivalent conductivity which can be taken from ${ }^{18}$, p. $465 . F$ is the Faraday constant. The following relation between $u$ and $\nu_{\mathrm{D}}$ results from Eq. (47), (48), (49) and (42) :

$$
u=\frac{1}{3} v_{\mathrm{D}} s \frac{2 p}{h v_{0}} \tanh \frac{h v_{0}}{2 k T} .
$$

Since the diffusion rate for structure migration is far more inaccurately known than $u$, Eq. (50) is used to calculate $v_{\mathrm{D}}$. The previously used values $v_{0}=10^{13} \sec ^{-1}$ and $p=2 \AA \cdot \frac{1}{2} e$ give

$$
v_{\mathrm{D}}=1.3 \times 10^{12} \mathrm{sec}^{-1} \text {. }
$$

This agrees well with what is expected according to EIGEN ${ }^{6}$ who has shown, by comparing correspond-

18 R. A. Robinson and R. H. Stokes, Electrolyte solutions, Butterworths, London 1959. ing investigations of ice and liquid water, that the proton transition in the liquid medium in the primary structure around $\mathrm{H}^{+}$, that is, the proton transition in the inner hydrogen bridges of grouping $\mathrm{H}_{5} \mathrm{O}_{2}{ }^{+}$or $\mathrm{H}_{9} \mathrm{O}_{4}{ }^{+}$proceeds $\mathrm{I}-2$ orders of magnitude more rapidly than the structure diffusion of these groups. The value of $\nu_{\mathrm{D}}$ is indeed approximately one order of magnitude smaller than $v_{0}$, the tunneling frequency of the excess proton in the hydrogen bridge of $\mathrm{H}_{5} \mathrm{O}_{2}{ }^{+}$or $\mathrm{H}_{9} \mathrm{O}_{4}{ }^{+}$.

This estimation has thus demonstrated that the small shift of the weights of the proton boundary structures due to the external field is the field-dependent mechanism of anomalous proton conductivity.

For fields of the order of $10^{6}-10^{7} \mathrm{Volt} / \mathrm{cm} \Delta G$ approximates 1 - corresponding to a complete displacement of the proton in the field direction. Such fields occur in electrode processes ${ }^{19}$ and especially in the membranes of biological systems. Thus it seems obvious that the great variation of $\Delta G$ by the electrical field, i. e. the extremely large polarizability of hydrogen bonds with symmetrical double minimum potential well is of decisive importance for the behavior of these systems.

We wish to thank Mr. H. Pfeiffer for the curve calculations. Our thanks are also due to the Deutsche Forschungsgemeinschaft for providing us with the means required for this work.

19 G. ErTel, Technische Hochschule Hannover, PhysikalischChemisches Institut (private communication). 